\title{
Topological Charges in Monopole Theories
}

\author{
P. A. Horváthy ${ }^{1}$ and J. H. Rawnsley ${ }^{2}$
}

1 Centre de Physique Théorique, CNRS, Luminy Case 907, F-13288 Marseille Cedex 9, France

2 Mathematics Institute, The University of Warwick, Coventry CV4 7AL, England

\begin{abstract}
Let us consider a monopole theory with a compact, simply connected gauge group and the Higgs field in the adjoint representation. Using root theory we show that.

(i) The homotopy class of the Higgs field is a $p$-tuple of integers where $p$ is the dimension of the centre of the residual symmetry group. These "Higgs charges" can be expressed as surface integrals of differential forms.

(ii) To any invariant polynomial on the Lie algebra is associated a topological invariant which turns out to be a combination of the Higgs charges.

(iii) Electric charge is quantized. The monopole's magnetic charge is a combination - with the Higgs charges as coefficients - of $p$ basic magnetic charges which satisfy generalized Dirac conditions.

The example of $G=\mathrm{SU}(N)$ is worked out in detail.
\end{abstract}

\section{Introduction}

The fundamental role played by topological invariants in monopole theory has been recognized since the very beginning $[1,2,3,18]$. Essentially three types of such "charges" have been considered so far:

(i) Assume that the full gauge group $G$ is broken spontaneously to $H$ by the vacuum expectation value of the Higgs field $\Phi$. The usual requirements concerning the asymptotic behaviour of $\Phi$ imply the existence of a map from $S^{2}$, the 2-sphere at infinity, into an orbit $G / H$ of $G$. We have thus a homotopy class

$$
[\Phi] \in \pi_{2}(G / H) \text {. }
$$

This first topological invariant shall be referred to as the Higgs charge.

(ii) Let $F$ denote the field strength tensor. If the Higgs field is covariantly constant, the quantity

$$
I=\int_{S^{2}} \operatorname{tr}(F \cdot \Phi)
$$


is also invariant under smooth deformations. It appears for instance in the formula of Bogomolny for the lower bound of the energy [4]. Remarkably, the integrand of this "trace invariant" (1.2) is just the topological charge density of the 4-dimensional pure Yang-Mills theory to which the static Yang-Mills system is equivalent if the Higgs potential vanishes [5].

(iii) To each generator of the unbroken symmetry group $H$ corresponds a massless gauge particle one of which is identified with the photon of electromagnetic interactions $[2,3]$. The possible electric charges are eigenvalues of the charge operator

$$
Q_{\mathrm{em}}=e_{0} \Phi /|\Phi| .
$$

The electromagnetic field itself is the projection of the gauge-field onto the Higgs-direction $[7,8,9]$. The monopole's magnetic charge is defined to be

$$
g=\left(1 / 4 \pi e_{0}\right) \int_{S^{2}} \operatorname{Tr}(F \cdot \Phi /|\Phi|) .
$$

These charges are of course intimately related. The aim of this paper is to give a systematic study of all the aformentioned topological invariants in a gauge theory with a compact, simply connected gauge group, broken spontaneously by a Higgs field in the adjoint representation.

We show first that the Higgs charge (1.1) is an $p$-tuple of integers

$$
\Phi=\left(m_{1}, \ldots, m_{p}\right),
$$

where $p$ is the dimension of $Z(\mathfrak{h})$, the centre of the Lie algebra of $H[6,9]$. The $m_{i}$ 's are also expressed as surface integrals

$$
m_{i}=(1 / 2 \pi \sqrt{-1}) \int_{S^{2}} \Phi^{*} \omega^{(i)} .
$$

The closed (but not exact) 2-forms $\omega^{(i)}$ on $G / H$ correspond with properly scaled vectors of a basis of $Z(\mathfrak{h})$.

Next we show that, for any invariant polynomial $f$ on the Lie algebra of $G$ which is homogeneous of order $k+1$ the quantity

$$
I^{(f)}=\int_{S^{2}} f(F, \Phi, \ldots, \Phi)
$$

is again a topological invariant, supposing that $D \Phi=0$. This is proved by showing that $I^{(f)}$ can be written, just like the Higgs charges in (1.6), as the integral of a closed 2 -form. Comparing the two formulae we see that $I^{(f)}$ is a linear combination of the Higgs charges:

$$
I(f)=2 \pi \sqrt{-1} \sum_{k=1}^{p} \lambda_{k} m_{k} .
$$

The coefficients $\lambda_{k}$ are explicitly obtained. They depend only on the modulus of the Higgs field and the group structure (see Sect. 3).

In particular, we give formulae for computing the "higher order invariants" [6] of the form

$$
f(F, \Phi, \ldots, \Phi)=\operatorname{tr}\left(F \cdot \Phi^{n}\right), \quad n=1,2, \ldots .
$$

Equation (1.8) generalizes the corresponding expression for $\mathrm{SU}(3)[10,11]$. 
We study then the electromagnetic properties. The electric charge operator is defined by the Higgs field $[8,13,14]$. In order to have quantized electric charge $\Phi$ must define a $\mathrm{U}(1)$ (rather then merely an $R$ ) subgroup. In this case there exists an (unique up to sign) minimal $\mathrm{U}(1)$ generator $\zeta_{0}$ pointing in the electromagnetic direction. This minimal generator plays an important role in the sequel. First:

All electric charges in the theory are integer multiples of

$$
q_{\min }=e_{0} /\left|\zeta_{0}\right| \text {. }
$$

$\zeta_{0}$ here is the minimal U(1) generator determined by the Higgs field's direction (see Sect. 5).

The magnetic charge $g$ of the monopole is in turn a combination - with $\zeta_{0^{-}}$ dependent coefficients - of $p$ partial magnetic charges $g_{1}, \ldots, g_{p}$ each of which is the multiple by the corresponding Higgs charge $m_{k}$ of a basic magnetic charge $g_{k}^{(0)}$ :

$$
g_{k}=m_{k} g_{k}^{(0)} \text {. }
$$

$q_{\min }$ and $g$ satisfy a generalized Dirac condition $[13,14]$.

The basic magnetic charges correspond to the "fundamental monopoles" in $[27,28]$.

The general theory is illustrated on the example of $G=\operatorname{SU}(N)[8,15,18]$.

We conclude that root theory $[16,17]$, combined with that of differential forms $[19,23]$ provides us with a via regia to solve any problem concerning topological invariants in monopole theory.

\section{Roots, Weights and Characters}

The basic facts and definitions of root theory we summarize here without proof will be used through the whole paper. For more details the reader should consult refs. $[16,17]$.

Let $G$ denote a compact, simply connected (and hence semisimple) Lie group having Lie algebra $\mathscr{G}$. Let us choose a point $\xi_{0}$ from $\mathscr{G}$, and denote by $H$ its stablizer under the adjoint action of $G$. The orbit of $\xi_{0}$ is identified with $G / H$.

Let us choose a maximal torus $T \subset G$ in such a way that $\mathscr{T}$, its Lie algebra, contains $\xi_{0}$. $T$ is a maximal torus also for $H$, and $\mathscr{T}^{C}$, the complexification of $\mathscr{T}$, is a Cartan subalgebra of $\mathscr{G}^{C}$.

Denote by $\Delta$ the set of roots. $\Delta \subset \sqrt{-1} \mathscr{T}^{*}$. Let $\Delta^{+}$be a positive root system and consider the simple roots

$$
\alpha_{1}, \ldots, \alpha_{r}(r=\operatorname{dim} T=\operatorname{rank} \text { of } G) .
$$

Define the subset of indices $I=\left\{i_{1}, \ldots, i_{p}\right\}$ by

$$
i_{k} \in I \quad \text { if } \quad \alpha_{i_{k}}\left(\xi_{0}\right) \neq 0 .
$$

$I$ is empty if and only if $\xi_{0}$ vanishes. Hence $I$ contains $p$ elements, $0<p<r$.

Let us choose the vectors $\xi_{1}, \ldots, \xi_{r}$ from $\mathscr{T}$ so that

$$
\alpha_{i}\left(\xi_{j}\right)=\sqrt{-1} \delta_{i j}, \quad i, j=1, \ldots, r .
$$

The $\xi_{j}$ 's form a basis of $\mathscr{T}$, dual to the $\alpha_{i}{ }^{\prime}$ s. Let $Z(\mathfrak{h})$ denote the centre of $\mathfrak{h}$, the Lie algebra of $H$. 


\section{Theorem 2.1.}

$$
\left\{\xi_{i_{1}}, \ldots, \xi_{i_{p}}\right\} \text { is a basis for } Z(\mathfrak{h}) \text {. }
$$

Consequently $p$ is the dimension of $Z(\mathfrak{h})$. Since $\xi_{0}$ is in the centre, it is a linear combination of the $\xi_{i_{k}}$ 's, $i_{k} \in I$ :

$$
\xi_{0}=\sum_{i_{k} \in I} a_{k} \xi_{i_{k}}
$$

where

$$
a_{k}=\alpha_{i_{k}}\left(\xi_{0}\right) / \sqrt{-1} .
$$

$G$ is semisimple and admits thus a negative definite $\mathrm{Killing}$ form $B(\cdot, \cdot)$. To each root $\alpha \in \Delta$ we may associate a unique $\eta_{\alpha} \in \mathscr{T}^{C}$ defined by

$$
B\left(\eta_{\alpha}, \zeta\right)=\alpha(\zeta), \quad \zeta \in \mathscr{T}^{C}
$$

If $\mu$ is a linear function on $\mathscr{T}^{C}$ with values in $C$, we may define tentatively a map $\chi$ from $T$ to $\mathrm{U}(1)$ by

$$
\chi(\exp \zeta)=\exp (\mu(\zeta)), \quad \zeta \in \mathscr{T} .
$$

$\chi$ is clearly a character of $T$ as soon as it is well-defined. This happens if for any root $\alpha \in \Delta$,

$$
2 \mu\left(\eta_{\alpha}\right) / \alpha\left(\eta_{\alpha}\right) \in Z \text {. }
$$

Any root being an integral combination of simple roots, it is enough to require (2.7) to hold for the simple roots. Let us define $\mu_{1}, \ldots, \mu_{r}$ by

$$
2 \mu_{i}\left(\eta_{\alpha_{j}}\right) / \alpha_{j}\left(\eta_{\alpha_{j}}\right)=\delta_{i j}
$$

Theorem 2.2. The $\mu_{i}$ 's form a Z-basis for those linear functions $\mu$ on $\mathscr{T}^{C}$ which exponentiate to characters.

These $\mu_{i}$ 's are the fundamental weights. Their associated characters are denoted by $\chi_{1}, \ldots, \chi_{r}$.

Every weight of any representation of $G$ is an integer combination of these fundamental weights and so the eigenvalues in any representation of an element $\xi$ of have the form

$$
\sum_{i=1}^{r} s_{i} \mu_{i}(\xi)
$$

Theorem 2.3. The character $\chi_{i}$ of $T$ extends to a character of $H$ if and only if $i=i_{k} \in I$. The extension is obtained at the Lie algebra level by setting $\mu_{i}=0$ on $\mathfrak{h} \cap \mathscr{T}^{\perp}$.

The characters are useful in describing the homotopy of $H$. Indeed, let $\gamma: S^{1}$ $\rightarrow H$ be a loop and consider a character $\chi$ of $H$. The composed map $\chi \circ \gamma$ goes from $S^{1}$ to U(1) and has thus a degree (or winding number) $m_{\chi}(\gamma) \in Z$. If $\gamma_{1}$ and $\gamma_{2}$ are homotopic loops in $H$, the degrees are the same since any smooth function with integer values is constant. So we get a homomorphism

$$
m_{\chi}: \pi_{1}(H) \rightarrow Z \text {. }
$$


The fundamental theorem of Bott [17] tells us now that we have enough characters to determine $\pi_{1}(H)$ completely:

Theorem 2.4. Consider those characters associated to the indices in I, and denote

$$
m_{k}(\gamma)=m_{\chi_{i_{k}}}(\gamma)
$$

The map

$$
\pi_{1}(H) \ni[\gamma] \rightarrow\left(m_{1}, \ldots, m_{p}\right) \in Z^{p}
$$

is an isomorphism.

If $\left(m_{1}, \ldots, m_{p}\right)$ is a $p$-tuple of integers, it is easy to construct a loop $\gamma$ having this $p$-tuple as its homotopy class. Indeed,

$$
\gamma(t)=\exp \left(2 \pi \sqrt{-1} t \sum_{i_{k} \in I} m_{k} \eta_{i_{k}}\right), \quad t \in[0,1]
$$

has this property. To see this let us apply the character $\chi_{i_{j}}$. We get

$$
\chi_{i_{j}}(\gamma(t))=\exp \left(2 \pi \sqrt{-1} t \sum m_{k} \mu_{i_{j}}\left(\eta_{i_{k}}\right)\right)=\exp \left(2 \pi \sqrt{-1} t m_{i_{j}}\right) .
$$

This map has clearly degree $m_{i^{\prime}}$.

We can switch from the $\mu_{i}^{\prime}$ 's to the $\alpha_{i}^{\prime}$ 's by the Cartan matrix $\left(C_{i j}\right)$ :

$$
\alpha_{i}=\sum_{j}^{r} C_{i j} \mu_{j} .
$$

$C_{i j}$ has integer entries. Set

$$
\eta_{i}=2 \eta_{\alpha_{i}} / \alpha_{i}\left(\eta_{\alpha_{i}}\right), \quad i=1, \ldots, r .
$$

The $\eta_{i}$ 's are dual to the $\mu_{j}^{\prime}$ 's:

$$
\mu_{i}\left(\eta_{j}\right)=\delta_{i j}
$$

Equation (2.13) implies

$$
\eta_{i}=(1 / \sqrt{-1}) \sum_{j}^{r} C_{j i} \xi_{j} .
$$

The $\mu_{j}^{\prime}$ s and $\xi_{j}$ 's are related in turn by

$$
\mu_{j}=\left(\alpha_{j}\left(\eta_{\alpha_{j}}\right) / 2 \sqrt{-1}\right) \cdot B\left(\xi_{j}, \ldots\right) .
$$

It follows that

$$
B\left(\xi_{i}, \eta_{j}\right)=\delta_{i j} \cdot\left(2 \sqrt{-1} / \alpha_{i}\left(\eta_{\alpha_{i}}\right)\right) .
$$

Observe that, according to (2.17), the $\mu_{i_{k}}$ 's $\left(i_{k} \in I\right)$ are represented by correctly scaled elements of the centre of $\mathfrak{h}$. The corresponding $\eta_{i_{k}}$ 's however do not belong necessarily to $Z(\mathfrak{h})$.

$\Gamma$, the unit lattice of $\mathscr{G}$, consists of those vectors $\zeta \in \mathscr{T}$ which satisfy

$$
\exp (2 \pi \zeta)=1 \text {. }
$$


Theorem 2.5. Any vector $\zeta$ of the unit lattice $\Gamma$ is a combination of $(\sqrt{-1}$ times $)$ the $\eta_{i}$ 's defined in (2.14) with integer coefficients:

$$
\zeta=\sqrt{-1} \sum_{i=1}^{r} n_{i} \eta_{i}
$$

The coefficients here are computed by

$$
n_{i}=\mu_{i}(\zeta) / \sqrt{-1}
$$

$\zeta_{0} \in \Gamma$ is said to be a minimal generator if $\exp \left(2 \pi \zeta_{0} t\right)=1$ the first time for $t=1$. This happens iff the integers $n_{i}$ in its expansion (2.20) have no common diviser. The set of minimal generators will be denoted by $\Gamma_{0}$.

If $\zeta \in \Gamma$, there exists a (unique up to sign) minimal generator parallel to it.

We illustrate the general theory on the example $G=\mathrm{SU}(N)$. We choose our maximal torus $T$ to consist of diagonal matrices in the standard representation.

$$
T=\left\{g=\operatorname{diag}\left(u_{1}, \ldots, u_{N}\right),\left|u_{i}\right|=1, \prod_{i}^{N} u_{i}=1\right\} .
$$

The rank of $\mathrm{SU}(N)$ is $(N-1)$.

For any diagonal matrix $a=\operatorname{diag}\left(a_{1}, \ldots, a_{N}\right)$, let $\varepsilon_{i}$ be the map giving its $i$ 's entry, $\varepsilon_{i}(a)=a_{i}$. Then $\left\{\varepsilon_{i}-\varepsilon_{j} \mid i \neq j\right\}$ gives a root system and $\left\{\varepsilon_{i}-\varepsilon_{j} \mid i<j\right\}$ a positive system whose simple roots are

$$
\alpha_{1}=\varepsilon_{1}-\varepsilon_{2}, \ldots, \alpha_{N-1}=\varepsilon_{N-1}-\varepsilon_{N} .
$$

The dual basis $\xi_{1}, \ldots, \xi_{N-1}$ reads

$$
\xi_{i}=(\sqrt{-1} / N)\left[\begin{array}{lllll}
N-i & & & & \\
& \cdot & N-i & & \\
& & & -i & \\
& & & & -i
\end{array}\right]\left\{\begin{array}{l}
i \\
N-i
\end{array} .\right.
$$

If we choose the trace form $\operatorname{Tr}$ (rather then the Killing form $B=-\operatorname{Tr} / N$ ) we get

$$
\eta_{\alpha_{i}}=\eta_{i}=\operatorname{diag}(0, \ldots, \stackrel{i}{i},-1,0, \ldots, 0)
$$

$\alpha_{i}\left(\eta_{\alpha_{i}}\right)=2$ in this case.

The fundamental weights are now

$$
\mu_{1}=\varepsilon_{1}, \mu_{2}=\varepsilon_{1}+\varepsilon_{2}, \ldots, \mu_{N-1}=\varepsilon_{1}+\ldots+\varepsilon_{N-1} .
$$

The associated fundamental characters are

$$
\chi_{j}(u)=\prod_{i=1}^{j} u_{i}, u=\operatorname{diag}\left(u_{i}\right) \in T, \quad j=1, \ldots, N-1 .
$$

Consider the base point

$$
\xi_{0}=\sqrt{-1} \operatorname{diag}\left(\lambda_{1}, \ldots, \lambda_{N}\right), \lambda_{j} \in R, \sum \lambda_{j}=0 .
$$


$\lambda_{1} \geqq \lambda_{2} \geqq \ldots$ can be assumed without loss of generality. (Such a situation can namely always be achieved by applying a Weyl transformation which corresponds physically to a constant gauge transformation.) $\xi_{0}$ is decomposed to blocks

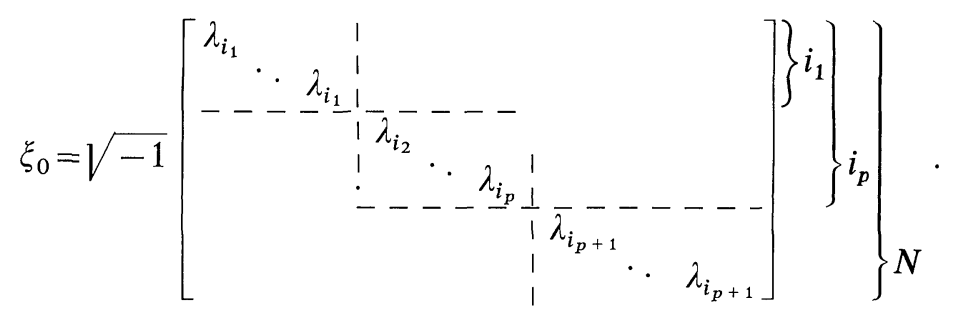

Observe that there are $(p+1)$ blocks. $p$ is hence, as anticipated by the notation, the dimension of the centre of $H \cdot Z(\mathfrak{h})$ is generated in fact by those $\xi_{i_{k}}$ 's, $i_{i} \in I$.

The little group is

$$
H=S\left\{\mathrm{U}\left(i_{1}\right) \times \mathrm{U}\left(i_{2}-i_{1}\right) \times \ldots \times \mathrm{U}\left(N-i_{p}\right)\right\} .
$$

Those characters belonging to the $i_{k}$ 's $\left(i_{k} \in I\right)$ extend to $H$. Denote $\operatorname{det}_{k}$ the determinant of the block between $i_{k}$ and $i_{k-1}$. Then

$$
\chi_{i_{k}}=\operatorname{det}_{1}(h) \ldots \operatorname{det}_{k}(h), \quad h \in H, k \leqq p .
$$

Let us fix $0<i_{1}<\ldots<i_{p}<i_{p+1}=N$. The dimension of the corresponding orbit $\mathcal{O}_{i_{1}, \ldots, i_{p}}$ is calculated by noting that the orbit is the same as it was for $\mathrm{U}(N)$. But for $\mathrm{U}(N)$ the stability group would be

$$
\mathrm{U}\left(i_{1}\right) \times \mathrm{U}\left(i_{2}-i_{1}\right) \times \ldots \mathrm{U}\left(N-i_{p}\right),
$$

so

$$
\operatorname{dim} \mathcal{O}_{i_{1}, \ldots, i_{p}}=2\left\{i_{1}\left(i_{2}-i_{1}\right)+\ldots+i_{p}\left(N-i_{p}\right)\right\}
$$

\section{The Higgs Charge}

The first of the topological charges we would like to study is the one associated to the way the gauge symmetry is broken by the vacuum expectation value of the Higgs field.

Let us consider in fact a Yang-Mills-Higgs theory in Minkowski space with a compact and simply connected gauge group $G$. The Higgs field $\Phi$ is assumed to be in the adjoint representation. We choose the standard Lagrangian

$$
-L=(1 / 4) F_{\mu \nu} F^{\mu \nu}+(1 / 2) D_{\mu} \Phi D^{\mu} \Phi+V(\Phi),
$$

where $F_{\mu \nu}=\partial_{\mu} A_{\nu}-\partial_{v} A_{\mu}+\left[A_{\mu}, A_{\nu}\right], D_{\mu} \Phi=\partial_{\mu} \Phi+\left[A_{\mu}, \Phi\right]$ (the fields are rescaled so that the coupling constant is 1 ).

Let us fix a gauge where $A_{0}$ is zero and suppose all the fields are static. We are interested in everywhere regular, finite energy (monopole) solutions. We assume, as usual, that the Higgs potential $V(\Phi)$ takes its minimum on an orbit of $G$. If $\xi_{0}=\Phi\left(x_{0}\right)$ is a reference point having $H$ as stability group, the orbit in question can be identified with the homogeneous space $G / H$. Assuming that the Higgs field takes its values asymptotically in $G / H$; we get a map (we denote also by $\Phi$ ) from $S^{2}$, the two-sphere at infinity, into the orbit $G / H$. $\Phi$ defines a class $[\Phi]$ in the second 
homotopy group of the orbit, and it is well-known that two situations are gaugerelated and are hence physically equivalent if the homotopy classes are the same [3]. In the sequel we shall call this class

$$
[\Phi] \in \pi_{2}(G / H)
$$

the Higgs charge of $\Phi$.

The aim of this section is to describe $\pi_{2}(G / H) \cdot \pi_{1}(G)=0$ by hypothesis and so $\pi_{2}(G)$ is also trivial. The long exact homotopy sequence implies that

$$
\delta: \pi_{2}(G / H) \rightarrow \pi_{1}(H)
$$

is an isomorphism. We describe it explicitly as follows: Denote by $U_{1}$ (respectively $U_{2}$ ) $S^{2} \backslash\{$ south pole $\}$ (respectively $S^{2} \backslash\{$ north pole $\}$ ). Let us choose a $\Phi$ representing a homotopy class in $\pi_{2}(G / H)$. Since the $U_{i}$ 's are contractible, $\Phi \mid U_{i}$, the restriction of $\Phi$ to $U_{i}$, lifts to a smooth map

$$
g_{i}: U_{i} \rightarrow G
$$

such that

$$
\Phi(x)=\operatorname{Ad}_{g_{i}} \xi_{0}, \quad x \in U_{i} .
$$

$g_{i}\left(x_{0}\right)=e$ can be assumed without loss of generality.

In $U_{1} \cap U_{2}, g_{1}$ and $g_{2}$ are related by

$$
g_{2}=g_{1} \cdot \gamma_{12} \text {. }
$$

$\gamma_{12}$ is smooth in $U_{1} \cap U_{2}$ and takes its values in $H$. Denote by $\gamma$ its restriction to $S^{1}$, the equatorial circle. $\gamma$ can be considered also as a loop in $H$ having a homotopy class $[\gamma] \in \pi_{1}(H)$. Furthermore, this homotopy class is the same for homotopic (in $\left.\pi_{2}(G / H)\right) \Phi$ 's. We define

$$
\delta([\Phi])=[\gamma] \in \pi_{1}(H) .
$$

Using Bott's theorem (Theorem 2.4) we get

Theorem 3.1. The Higgs charge of $\Phi$ is a p-tuple of integers,

$$
[\Phi]=\left(m_{1}, \ldots, m_{p}\right) \in Z^{p},
$$

where $m_{k}$ is the degree of the map

$$
\chi_{i_{k}}(\delta(\Phi)): S^{1} \rightarrow \mathrm{U}(1)
$$

The degree of a map $f: S^{1} \rightarrow U(1)$ can also be calculated by a contour integral viewing $\mathrm{U}(1) \subset C$ and $f$ as a contour:

$$
\operatorname{deg} f=(1 / 2 \pi \sqrt{-1}) \int_{f} d \chi / \chi
$$

Applied to $f=\chi(\gamma)$

$$
m_{\chi}(\gamma)=(1 / 2 \pi \sqrt{-1}) \int_{\gamma} d \chi / \chi
$$

The Higgs charges can be expressed also as integrals on the two-sphere at infinity of suitable closed (but not exact) 2 -forms $[6,9]$. To see this observe that 
each $\varrho \in Z(\mathfrak{h})$ determines an invariant 2 -form $\omega^{\varrho}$ on the orbit $G / H$. By invariance it is enough to define it at $\xi_{0}$ :

$$
\omega_{\xi_{0}} \varrho\left(\eta_{\xi_{0}}, \zeta_{\xi_{0}}\right)=B(\varrho,[\zeta, \eta]), \quad \zeta, \eta \in \mathscr{G} .
$$

$\omega^{\varrho}$ is a closed 2-form on $G / H$. Its pullback by $\Phi$ is thus a closed 2-form on $S^{2}$.

Theorem 3.2. Let us consider the two-forms

$$
\omega^{(k)}=\left\{\alpha_{i_{k}}\left(\eta_{\alpha_{i_{k}}}\right) / 2 \sqrt{-1}\right\} \cdot \omega^{\xi_{i}} k, \quad i_{k} \in I .
$$

The Higgs charges can be calculated as

$$
m_{i}(\Phi)=(1 / 2 \pi \sqrt{-1}) \int_{S^{2}} \Phi^{*} \omega^{(k)}, \quad i_{k} \in I .
$$

Proof. Let us define $U_{1}^{\varepsilon}$ and $U_{2}^{\varepsilon}$ respectively as the upper (respectively lower) part of $S^{2}$ between $0<\theta<\pi / 2+\varepsilon$ (respectively $\pi / 2-\varepsilon<\theta<\pi$ ). Their intersection is a narrow "collar" of width $2 \varepsilon$ containing the equator. Let $\varrho \in Z(\mathfrak{h})$ be arbitrary. Then

$$
\int_{S^{2}} \Phi^{*} \omega^{\varrho}=\lim _{\varepsilon \rightarrow 0} \int_{U_{1}^{\varepsilon}} \Phi^{*} \omega^{\varrho}+\int_{U_{2}^{\varepsilon}} \Phi^{*} \omega^{\varrho} .
$$

Let us choose lifts $g_{i}$ in $U_{i}^{\varepsilon}$ as in (3.5). Then we have the commuting diagram

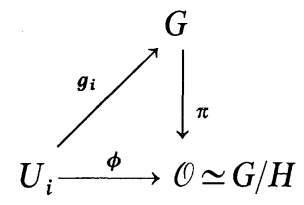

so

$$
\Phi^{*} \omega^{\varrho}=\left(\pi \circ g_{i}\right)^{*} \cdot \omega^{\varrho}=g_{i}^{*} \pi^{*} \omega^{\varrho} .
$$

$\pi^{*} \omega^{\varrho}$ is the (unique) extension to $G$ as a left-invariant 2 -form whose value at $e \in G$ is $B(\varrho,[\zeta, \eta])$. This 2 -form is exact, since $H^{2}(\mathscr{G} ; R)=0$. It is in fact the exterior derivative of the left-invariant 1 -form $\theta^{\circ}$ whose value at $e \in G$ is given by

$$
\theta_{e}^{\varrho}(\eta)=B(\varrho, \eta), \quad \eta \in \mathscr{G} .
$$

It is easy to see that

$$
\theta^{\varrho}=B(\varrho, \theta),
$$

where $\theta=g^{-1} d g$ is the Maurer-Cartan 1-form on $G$ [19].

$\pi^{*} \omega^{\varrho}=d \theta^{\varrho}$ and thus

$$
\Phi^{*} \omega^{\varrho}=g_{i}^{*} d \theta^{\varrho}=d\left(g_{i}^{*} \theta^{\varrho}\right)=d\left(B\left(\varrho, g_{i}^{-1} d g_{i}\right)\right) .
$$

But on the equator $g_{2}(x)=g_{1}(x) \cdot \gamma(x)$ and so, taking the respective orientations into account, we get

$$
\int_{S^{2}} \Phi^{*} \omega^{\varrho}=\int_{S^{1}} B\left(\varrho, \gamma^{-1} d \gamma\right)=\int_{\gamma} \theta^{\varrho}
$$

In particular, for

$$
\begin{gathered}
\varrho_{k}=\left\{\alpha_{i_{k}}\left(\eta_{\alpha_{i_{k}}}\right) / 2 \sqrt{-1}\right\} \cdot \xi_{i_{k}} \quad\left(i_{k} \in I\right), \\
B\left(\varrho_{k}, \cdot\right)=\mu_{i_{k}}=d \chi_{i_{k}} .
\end{gathered}
$$


So

$$
\int_{S^{2}} \Phi^{*} \omega^{(k)}=\int_{\gamma} \theta^{(k)}=\int_{\gamma} d \chi_{i_{k}} / \chi_{i_{k}}=2 \pi \sqrt{-1} m_{k}(\Phi)
$$

Q.E.D.

Note for further reference that for any $\varrho \in Z(\mathfrak{h})$

$$
\Phi^{*} \omega^{\varrho}=2 \pi \sqrt{-1} \sum_{i_{k} \in I} m_{k}(\Phi) B\left(\eta_{k}, \varrho\right) .
$$

Remark 3.3. The 2 -forms $\omega^{\varrho}$ on $\mathcal{O}_{\xi_{0}}$ we associated to the elements $\varrho \in Z(\mathfrak{h})$ are closely related to the coadjoint orbit construction of Kirillov, Kostant and Souriau [21-23]. Observe in fact that $\mathcal{O}_{\xi_{0}}$ projects onto $\mathcal{O}_{\varrho}$, the orbit of $\varrho$. Indeed, $\eta=\operatorname{Ad}_{g} \xi_{0} \in \mathcal{O}_{\xi}$ is mapped to $\pi_{\varrho}(\eta)=\operatorname{Ad}_{g} \varrho$. It follows from $\varrho \in Z(\mathfrak{h})$ that $\pi_{\varrho}$ is welldefined.

$\mathscr{G}$ can be identified with $\mathscr{G}^{*}$, its algebraic dual, through the Killing form, so $\mathcal{O}_{\varrho}$ can alternatively be considered as a coadjoint orbit, carrying a natural symplectic form. This is defined as the invariant extension to $\varrho$ of

$$
\Omega_{\varrho}^{(\varrho)}\left(\eta_{\varrho}, \zeta_{\varrho}\right)=B(\varrho,[\eta, \zeta]), \quad \eta, \zeta \in \mathscr{G} .
$$

Plainly,

$$
\omega^{\varrho}=\pi_{\varrho}^{*} \Omega^{\varrho} .
$$

Observe that, according to (3.13), the 2 -form $\Omega^{\circ}$ must be integral which means that, for any 2 -sphere lying in $\mathcal{O}_{Q}$,

$$
(1 / 2 \pi \sqrt{-1}) \int_{S^{2}} \Omega^{e} \in Z
$$

In other words the orbit $\mathcal{O}_{\varrho}$ endowed with its canonical symplectic structure is prequantizable.

Coadjoint orbits have been studied extensively in the literature [21-24]. Their knowledge makes it easy to describe the Higgs charges (see Sect. 7).

Remark 3.4. The construction of the isomorphism $\delta: \pi_{2}(G / H) \rightarrow \pi_{1}(H)$ we gave here differs slightly from the usual procedure $[8,14,18]$. It is not difficult to show however that our loop $\gamma$ and the standard one are homotopic.

\section{Generalized Invariants}

At the "Prasad-Sommerfield limit" of vanishing Higgs potential [25] the static Yang-Mills Higgs system becomes equivalent to a pure Yang-Mills system [5]. To the 4-dimensional topological charge corresponds the quantity

$$
I=\int_{S^{2}} B(F, \Phi) .
$$

To the self-duality equations in 4 dimensions correspond the equations of Bogomolny [4] whose solutions have, for a fixed value of $I$, minimal energy. Furthermore, this energy is proportional to $I$.

Recently [6] (4.1) has been generalized to

$$
I^{(n)}=\int_{S^{2}} \operatorname{tr}\left(F \Phi^{n}\right)
$$


where $\Phi$ is in some matrix representation so it is meaningful to speak of its $n^{\text {th }}$ power.

In this section we and

(i) further generalize (4.1) and (4.2) field.

(ii) relate them to the Higgs charges and to other characteristics of the Higgs

In fact we associate a topological invariant to any invariant polynomial on the Lie algebra of $G$.

A polynomial is a sum of homogenous terms so it is enough to restrict our attention to homogenous invariant polynomials. Remember that an invariant polynomial of order $n$ is a function $f: \mathscr{G} \rightarrow R$ such that for any $t \in R$ and $\zeta \in \mathscr{G}$,

$$
f(t \zeta)=t^{n} f(\zeta)
$$

and

$$
f\left(\operatorname{Ad}_{g} \zeta\right)=f(\zeta)
$$

Alternatively, we can view $f$ as an invariant $n$-linear function on the Lie algebra obtained as

$$
f\left(\zeta_{1}, \ldots, \zeta_{n}\right)=\frac{1}{n !} \frac{\partial^{n} f\left(t_{1} \zeta_{1}+\ldots t_{n} \zeta_{n}\right)}{\partial t_{1} \ldots \partial t_{n}}
$$

This $f$ satisfies

$$
f\left(\left[\zeta, \zeta_{1}\right], \ldots, \zeta_{n}\right)+\ldots+f\left(\zeta_{1}, \ldots,\left[\zeta, \zeta_{n}\right]\right)=0 .
$$

Let us now consider a static Yang-Mills system $\left(A_{\mu}, \Phi\right)$ and assume (to have finite energy) that the Higgs potential is asymptotically covariantly constant:

$$
D_{\mu} \Phi=0 \text {. }
$$

We intend to show

Theorem 4.1. Let $f$ be an invariant $(n+1)$-linear function on the Lie algebra of $G_{j}$

$$
I^{(f)}=\int_{S^{2}} f(F, \Phi, \ldots, \Phi)
$$

is a topological invariant, actually independent of $A$ as long as $D_{\mu} \Phi=0$.

Theorem 4.1 is proved by relating (4.8) to integrals of 2-forms on the twosphere at infinity. Let us define in fact

$$
\omega_{\xi}^{(f)}\left(\eta_{\xi}, \zeta_{\xi}\right)=f([\zeta, \eta], \xi, \ldots, \xi), \quad \xi \in G / H .
$$

That $\omega^{(f)}$ is a smooth, $G$-invariant, closed 2-form on $G / H$ is verified in the standard way.

Similarly, let us define the 1 -form $\theta^{\left(f, \xi_{0}\right)}$ on $G$ as the left-invariant extension of

$$
\eta \rightarrow f\left(\eta, \xi_{0}, \ldots, \xi_{0}\right) \text {. }
$$

Clearly,

$$
\theta^{\left(f, \xi_{0}\right)}=f\left(g^{-1} d g, \xi_{0}, \ldots, \xi_{0}\right)
$$


If $\pi$ is the projection $G \rightarrow G / H$ defined by $\pi(g)=\operatorname{Ad}_{g} \xi_{0}$, then

$$
\pi^{*} \omega^{(f)}=d \theta^{\left(f, \xi_{0}\right)} .
$$

Let us now calculate $\Phi^{*} \omega^{(f)}$. $\Phi$ lifts locally to a map $g: U \rightarrow G$ so that $\Phi(x)=\pi(g(x))$.

$$
\Phi^{*} \omega^{(f)}=g^{*} \pi^{*} \omega^{(f)}=g^{*} d \theta^{\left(f, \xi_{0}\right)}=d\left(g^{*} \theta^{\left(f, \xi_{0}\right)}\right)
$$

Consider

$$
a=\operatorname{Ad}_{g^{-1}} A+g^{-1} d g .
$$

It follows from $D_{\mu} \Phi=0$ that $a$ takes its values in the Lie algebra of $H$. Indeed, $\left[a, \xi_{0}\right]=\left[\operatorname{Ad}_{g^{-1}} A, \xi_{0}\right]+\left[g^{-1} d g, \xi_{0}\right]=g^{-1}\left([A, \Phi]+d\left(\operatorname{Ad}_{g} \xi_{0}\right)\right) g=g^{-1}(D \Phi) g=0$.

On the other hand,

$$
d a+(1 / 2)[a \wedge a]=\operatorname{Ad}_{g^{-1}} F
$$

so using the invariance of $f$,

$$
g^{*} \theta^{\left(f, \xi_{0}\right)}=f\left(a, \xi_{0}, \ldots, \xi_{0}\right)-f(A, \Phi, \ldots, \Phi) .
$$

Hence by (4.13)

$$
\Phi^{*} \omega^{(f)}=f\left(d a, \xi_{0}, \ldots, \xi_{0}\right)-d(f(A, \Phi, \ldots, \Phi)) .
$$

\section{Lemma 4.2.}

$$
f\left([\mathfrak{h}, \mathscr{G}], \xi_{0}, \ldots, \xi_{0}\right)=0 .
$$

Indeed, it follows from (4.6) that

$$
f\left([\mathfrak{h}, \mathscr{G}], \xi_{0}, \ldots, \xi_{0}\right)+n \cdot f\left(\mathscr{G},\left[\mathfrak{h}, \xi_{0}\right], \ldots, \xi_{0}\right)=0,
$$

but $\left[\mathfrak{h}, \xi_{0}\right]=0$ by the definition of $\mathfrak{h}$.

It follows now from (4.13), (4.15) and the invariance of $f$ that the first term in (4.14) is just $f\left(d a, \xi_{0}, \ldots, \xi_{0}\right)=f(F, \Phi, \ldots, \Phi)$. We conclude that

$$
\Phi^{*} \omega^{(f)}=f(F, \Phi, \ldots, \Phi)-d\{f(A, \Phi, \ldots, \Phi)\} .
$$

What we have proved is the following:

Theorem 4.3. Equation (4.8) is expressed as a surface integral at infinity:

$$
I^{(f)}=\int_{S^{2}} \Phi^{*} \omega^{(f)} .
$$

Theorem 4.1 is proved now by noting that the integral (4.17) depends only on the homotopy class of $\Phi$ in $\pi_{2}(G / H)$. It is also independent of $A$.

Having expressed $I^{(f)}$ as the integral of a 2 -form, it is easy to relate it to the Higgs charges. The point is that $\omega^{(f)}$ is just $\omega^{\varrho}$ (Definition (3.11)) for a suitable $\varrho \in Z(\mathfrak{h})$. To see this observe that $\eta \rightarrow f\left(\eta, \xi_{0}, \ldots, \xi_{0}\right)$ is a linear function on $\mathscr{G}$ and thus, by the non-degeneracy of the Killing-form $B$, there exists a unique $\varrho \in \mathscr{G}$ such that

$$
f\left(\eta, \xi_{0}, \ldots, \xi_{0}\right)=B(\varrho, \eta), \quad \eta \in \mathscr{G}
$$


From (4.15) we get on the other hand

$$
0=B(\varrho,[\mathscr{G}, \mathfrak{h}])=B([\mathfrak{h}, \varrho], \mathscr{G}),
$$

so $\varrho \in Z(\mathfrak{h})$. Comparing (4.9) and (3.21) we conclude

Proposition 4.4. $\omega^{(f)}=\omega^{\varrho}$.

To find $\varrho$ explicitly expand it as

$$
\varrho=\sum_{i_{k} \in I} b_{k} \xi_{i_{k}}
$$

( $\varrho$ is in the centre!). Using (4.18) and (2.14) we get

$$
\varrho=\sum_{i_{k}}\left\{\left[\alpha_{i_{k}}\left(\eta_{\alpha_{i_{k}}}\right) / 2 \sqrt{-1}\right] \cdot f\left(\eta_{i_{k}}, \xi_{0}, \ldots, \xi_{0}\right)\right\} \cdot \xi_{i_{k}} .
$$

Using (3.21) this implies

$$
\Phi^{*} \omega^{\varrho}=2 \pi \sqrt{-1} \sum_{i_{k}} f\left(\eta_{i_{k}}, \xi_{0}, \ldots, \xi_{0}\right) \Phi^{*} \omega^{(k)}
$$

Comparing to (3.12) we conclude

Theorem 4.5. The topological invariant $I^{(f)}$ is a linear combination of the Higgs charges $m_{k}\left(i_{k} \in I\right)$,

$$
I^{(f)}=2 \pi \sqrt{-1} \sum_{i_{k} \in I} \lambda_{k}^{(f)} m_{k}
$$

where the coefficients are given as

$$
\lambda_{k}^{(f)}=f\left(\eta_{i_{k}}, \xi_{0}, \ldots, \xi_{0}\right) .
$$

This works in particular for $f(F, \Phi)=B(F, \Phi)$ :

Corollary 4.6. The trace-(or rather Killing) invariant (4.1) is expressed as

$$
I=2 \pi \sqrt{-1} \sum_{i_{k}} \lambda_{k}^{(B)} m_{k}
$$

where

$$
\lambda_{k}^{(B)}=B\left(\eta_{i_{k}}, \xi_{0}\right)=\left\{2 \sqrt{-1} / \alpha_{i_{k}}\left(\eta_{\alpha_{k}}\right)\right\} \cdot \alpha_{i_{k}}\left(\xi_{0}\right) .
$$

Equations (4.23) $\cdot(4.24)$ generalize the formula known for $\mathrm{SU}(3)[10,11]$.

Even better, it applies to the higher-order invariants (4.2). Indeed, choosing a matrix-representation for the Lie algebra $\Phi^{n}$, the $n^{\text {th }}$ power of $\Phi$, becomes meaningful and we can consider

$$
f(F, \Phi, \ldots, \Phi)=B\left(F, \Phi^{n}\right) .
$$

$\Phi$ appears $n$ times in the argument so $f$ is an $(n+1)$-linear function. Equation (4.25) is also invariant, so Theorem 4.4 yields

Corollary 4.7. $I^{(n)}=B\left(F, \Phi^{n}\right)$ is a topological invariant. It is a linear combination of the Higgs charges,

$$
I^{(n)}=2 \pi \sqrt{-1} \sum_{i_{k} \in I} \lambda_{k} \cdot m_{k}
$$


where the coefficients are given by

$$
\lambda_{k}=B\left(\eta_{i_{k}}, \xi_{0}^{n}\right)
$$

\section{Electromagnetic Charges}

The last charges we want to study are those associated to the electromagnetic properties. Indeed, it is a fundamental requirement that the residual symmetry group contains the electromagnetic gauge group $\mathrm{U}(1)$ as a subgroup. The electromagnetic direction must be defined at every point. This can be done in a gauge-invariant way by a Higgs field.

Indeed, let us define the electromagnetic field as the projection onto the Higgs field's direction of the gauge field:

$$
\mathscr{F}=\left(1 / e_{0}\right) B(F, \Phi /|\Phi|) .
$$

( $D \Phi=0$ on $S^{2}$ implies that the definition of the electromagnetic field is unambigous $[7-9,18]$.)

According to general principles [26], the elementary particles appearing in the theory are multiplets belonging to some irreducible representation of the gauge group. The possible electric charges are the eigenvalues of the electric charge operator

$$
Q_{\mathrm{em}}=e_{0} \Phi /|\Phi|
$$

The electric charges observed in nature are quantized so $Q_{\mathrm{em}}$ must generate a $\mathrm{U}(1)$ rather then merely an $R$-subgroup in $H[12,13]$. This happens if a suitable real multiple of $\xi_{0}$ belongs to $\Gamma$, the unit lattice (see Sect. 2.) If so, there exists an (up to sign unique) minimal generator $\zeta_{0}$ parallel to $\xi_{0}$. Hence by $(2.20)$

$$
\zeta_{0}=\sqrt{-1} \sum_{i}^{r} n_{i} \eta_{i}, \quad n_{i} \in Z,
$$

where the coefficients have no common divisor.

It is easy to see what condition must $\xi_{0}$ satisfy for this. $\xi_{0}$ is in the centre so

$$
\xi_{0}=\sum_{i_{k} \in 1} a_{k} \xi_{i_{k}}
$$

Inverting (2.16) we express $\xi_{i_{k}}$ in terms of the $\eta_{i}$ 's and the Cartan matrix $C_{i j}$ :

$$
\xi_{i_{k}}=\sqrt{-1} \sum_{j}^{r}\left(C^{-1}\right)_{i_{k j}} \eta_{j}
$$

But [16]

$$
\left(C^{-1}\right)_{i j}=(\operatorname{adj} C)_{i j} / \operatorname{det} C=(\operatorname{adj} C)_{i j} /|Z(G)|,
$$

since $\operatorname{det} C=|Z(G)|$, the number of elements in the centre of $G$. Thus

$$
\xi_{0}=(\sqrt{-1} /|Z(G)|) \sum_{j}^{r}\left\{\sum_{i_{k} \in I} a_{k}(\operatorname{adj} C)_{i_{k} j}\right\} \eta_{j} .
$$

$(\operatorname{adj} C)_{i j}$ and $|Z(G)|$ are integers so 
Proposition 5.1. $\xi_{0}$ generates $a \mathrm{U}(1)$ if and only if its coefficients $a_{k}$ in the expansion (5.4) are rationally related.

In what follows we shall assume $\xi_{0}$ has this property. Let us choose a minimal generator $\zeta_{0}$ parallel to $\xi_{0}$ and consider the new Higgs field $\Psi(x)$ obtained from $\Phi(x)$ by rescaling:

$$
\Psi(x)=\left(\left|\zeta_{0}\right| /\left|\xi_{0}\right|\right) \Phi(x)
$$

$|\Psi(x)|=\left|\zeta_{0}\right|$, so $Q_{\mathrm{em}}$ reads also

$$
Q_{\mathrm{em}}=e_{0} \cdot \Psi(x) /\left|\zeta_{0}\right| .
$$

(This apparently complicated procedure is needed since the only coherent way to define the electric charge operator is through a Higgs field.)

Representation theory $[16,17]$ - see Sect. 2 - tells us that in any irreducible representation the eigenvalues of $Q_{\mathrm{em}}$ belong to the weight lattice: there exist integer coefficients $k_{i}$ such that

$$
q=\sum k_{i} \mu_{i}\left(Q_{\mathrm{em}}\right) .
$$

Using the expansion (5.3) and the duality property (2.15) it is easy to evaluate $q$ :

\section{Theorem 5.2.}

(i) Electric charge is quantized:

$$
q=\left(\sum_{i=1}^{r} k_{i} n_{i}\right) q_{\min } .
$$

(ii) The minimal charge $q_{\min }$ is determined by the length of the minimal generator $\zeta_{0}$

$$
q_{\min }=e_{0} /\left|\zeta_{0}\right|
$$

The minimal charge $q_{\min }$ is attained in some representation: the $k_{i}$ 's can be chosen to satisfy $\sum k_{i} n_{i}=1$ since the $n_{i}$ 's have no common factor.

$\zeta_{0}$ belongs also to the centre and so

$$
\zeta_{0}=\sum b_{k} \cdot \xi_{i_{k}}
$$

Thus

$$
\left|\zeta_{0}\right|^{2}=B\left(\zeta_{0}, \zeta_{0}\right)=\sum \sum \sqrt{-1} n_{j} b_{k} B\left(\eta_{j} \cdot \xi_{i_{k}}\right)=\sum\left\{2 / \alpha_{i_{k}}\left(\eta_{\alpha_{i_{k}}}\right)\right\} \cdot b_{k} n_{i_{k}}
$$

So the minimal charge reads

$$
q_{\min }=e_{0}\left|\sum_{i_{k} \in I}\left\{2 / \alpha_{i_{k}}\left(\eta_{\alpha_{i_{k}}}\right)\right\} \cdot n_{i_{k}} b_{k}\right|^{-1 / 2} .
$$

On the other hand, monopoles carry also a magnetic charge defined as

$$
g=(1 / 4 \pi) \int_{S^{2}} \mathscr{F} \text {. }
$$

Using the definition (5.1) of the electromagnetic field and the fact that on $S^{2}|\Phi|=\left|\xi_{0}\right|,(5.14)$ becomes

$$
g=\left(1 / 4 \pi e_{0}\left|\xi_{0}\right|\right) \int_{S^{2}} B(F, \Phi)
$$


Here we recognize the trace invariant - topological charge of Sect. 4. Consequently:

Corollary 5.3. The magnetic charge (5.14) is proportional to the topological charge (or, more exactly, to the trace invariant) $I$ :

$$
g=\left(1 / 4 \pi e_{0}\left|\xi_{0}\right|\right) I(\Phi)=\left(1 / 4 \pi e_{0}\left|\zeta_{0}\right|\right) I(\Psi)
$$

Equation (5.16) can be further developed using the results of Sect. 4.

$$
\begin{aligned}
g & =\left(1 / 2 e_{0}\right) \sum_{i_{k} \in I} m_{k} \sqrt{-1} B\left(\eta_{i_{k}}, \xi_{0} /\left|\xi_{0}\right|\right) \\
& =\left(1 / 2 e_{0}\right) \sum_{i_{k} \in I} m_{k} \sqrt{-1} B\left(\eta_{i_{k}}, \zeta_{0} /\left|\zeta_{0}\right|\right) \\
& =\left(1 / 2 e_{0}\right) \sum_{i_{k} \in I} m_{k}\left\{2 / \alpha_{i_{k}}\left(\eta_{\alpha_{i_{k}}}\right)\right\} \cdot b_{k} /\left|\zeta_{0}\right|
\end{aligned}
$$

Notice that $b_{k} /\left|\zeta_{0}\right|=a_{k} /\left|\xi_{0}\right|$. Finally we get:

Theorem 5.4. The magnetic charge of the monopole is a combination of $p$ "partial magnetic charges" $g_{1}, \ldots, g_{p}$ :

$$
g=\sum a_{k}\left(\left|\xi_{i_{k}}\right| /\left|\xi_{0}\right|\right) g_{k}=\sum b_{k}\left(\left|\xi_{i_{k}}\right| /\left|\zeta_{0}\right|\right) g_{k},
$$

where each of the $g_{k}$ 's is the multiple by $m_{k}$ of a fundamental charge $g_{k}^{(0)}$ :

$$
\begin{gathered}
g_{k}=m_{k} \cdot g_{k}^{(0)}, \\
g_{k}^{(0)}=\frac{1}{2 e_{0}\left|\xi_{i_{k}}\right|}\left[\frac{2}{\alpha_{i_{k}}\left(\eta_{\alpha_{i_{k}}}\right)}\right], \quad(k=1, \ldots, p) .
\end{gathered}
$$

How are the electric - and magnetic charges related? (5.9) and (5.19) imply that

$$
2 g_{\min } g_{k}=\left[2 / \alpha_{i_{k}}\left(\eta_{\alpha_{i_{k}}}\right)\right] /\left|\zeta_{0}\right|\left|\xi_{i_{k}}\right|
$$

so we conclude:

Theorem 5.5. The total magnetic charge $g$ and the minimal electric charge $q_{\min }$ satisfy a generalized Dirac condition:

$$
2 q_{\min } g=\sum_{i_{k} \in I}\left|\frac{2 m_{k} a_{k}}{\alpha_{i_{k}}\left(\eta_{\alpha_{i_{k}}}\right)}\right| / \sum_{i_{j} \in I}\left|\frac{2 n_{i_{j}} a_{j}}{\alpha_{i_{j}}\left(\eta_{\alpha_{i_{j}}}\right)}\right| .
$$

It is interesting to notice that the magnetic charge admits also a cohomological expression, i.e. can be written as the surface integral of a 2-form. Indeed, from (5.15) we get, using (3.21), that

$$
g=\sum_{i_{k} \in I}\left(a_{k}\left|\xi_{i_{k}}\right| /\left|\xi_{0}\right|\right) \frac{1}{2 e_{0}\left|\xi_{i_{k}}\right|} \int_{S^{2}} \Phi^{*} \omega^{\xi_{i_{k}}}
$$

comparing to (5.19):

$$
g_{k}=\frac{1}{2 e_{0}\left|\xi_{i_{k}}\right|} \int_{S^{2}} \Phi^{*} \omega^{\xi_{i_{k}}} .
$$

We see that the "basic charges" $g_{k}^{(0)}$ are associated with the "basic 2-forms" $\omega^{\left(\xi_{i_{k}}\right)}$ of Sect. 3 [9]. 
The situation is particularly simple if $\xi_{0}$ is parallel to one of the fundamental $\xi_{i}$ 's - say to $\xi_{k}$. Then the centre of $\mathfrak{h}$ is 1 -dimensional [13]. The Lie algebra of $H$ is decomposed now as

$$
\mathfrak{h}=u(1)+\mathscr{K},
$$

where $\mathscr{K}=[\mathfrak{h}, \mathfrak{h}]$ is semisimple. Physically, this corresponds to the situation $[13,14]$ when the residual symmetry group is (locally) the product of an electromagnetic $\mathrm{U}(1)$ and a color group $K$.

$I=\{k\}$, so we have just one Higgs charge $m=m_{k}$. The minimal electric charge reads now

$$
q_{\min }=e_{0}\left\{\alpha_{k}\left(\eta_{\alpha_{k}}\right) / 2 n_{k} b_{k}\right\}^{1 / 2} .
$$

There exists one basic magnetic charge, $g_{k}^{(0)}=g_{\min }$ and any magnetic charge is the multiple of it by the Higgs charge:

$$
g=m \cdot g_{\min }
$$

with

$$
g_{\min }=\left(1 / 2 e_{0}\right)\left\{2 b_{k} / \alpha_{k}\left(\eta_{\alpha_{k}}\right) n_{k}\right\}^{1 / 2} .
$$

(This follows also from (5.22) and (3.12).)

The generalized Dirac condition becomes

$$
2 q_{\min } \cdot g_{\min }=1 / n_{k} .
$$

The integer $n_{k}$ here counts the number of times the U(1) - and the semisimple subgroups intersect. Indeed, $H$ has the form [13]

$$
H=[\mathrm{U}(1) \times K] / Z,
$$

where $Z=\mathrm{U}(1) \cap K . Z \subset Z(K)$ so it has a finite number of elements. Equation (5.29) shows also that $n_{k}$ is the number of times $\mathrm{U}(1) \times K$ covers $H[15,18]$. Or this number is recovered by applying the weight $\mu_{i_{k}}$ to the generator of the U(1) subgroup of $H$, which is $\zeta_{0}$.

Notice that the minimal magnetic charge is - up to normalisation - the reciprocal of the length of the fundamental $\xi_{k}$ to which $\xi_{0}$ is parallel [14]:

$$
g_{\min }=\left\{e_{0} \alpha_{k}\left(\eta_{\alpha_{k}}\right)\left|\xi_{k}\right|\right\}^{-1}
$$

This follows from (5.19) noting that $\zeta_{0}=b_{k} \xi_{k}$ now.

\section{Application: $G=\mathrm{SU}(N)$}

The aim of this last section is to illustrate the general theory outlined above on the example of $G=\mathrm{SU}(U)$. This is also of practical interest, since most of the gauge groups considered in physics belong to this class.

$G=\mathrm{SU}(2)$. Let us start, in order to get a feeling what is going on, with $G=\mathrm{SU}(2)$. The base point now has the form

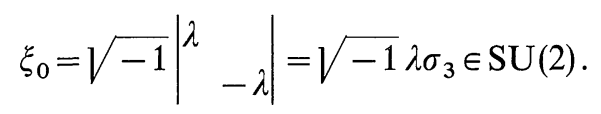


Identifying $\mathrm{SU}(2)$ with $R^{3}$, the orbit of $\xi_{0}$ will be a 2 -sphere of radius $|2 \lambda|$. The little group consists of the rotations around the third axis:

$$
H=\left\{\exp \left(2 \pi \sqrt{-1} \sigma_{3} t\right), t \in[0,1]\right\} .
$$

$I=\{1\}$ so $Z(\mathfrak{h}) \simeq \mathfrak{h} \simeq u(1)$ is generated by

$$
\xi_{1}=\sqrt{-1} \sigma_{3} / 2 \text {. }
$$

There is just one Higgs charge $m=m_{1}$. It is computed, according to Theorem 3.2 , by

$$
m(\Phi)=(1 / 2 \pi \sqrt{-1}) \int_{S^{2}} \Phi^{*} \omega
$$

where

$$
\omega=\omega^{(1)}=\omega^{\xi_{1}} / \sqrt{-1} .
$$

Observe that the orbit of $\xi_{0}$ - the 2 -sphere of radius $|2 \lambda|$-projects to the orbit of $\xi_{1}$ - the sphere of radius $1 / \sqrt{2}$,

$$
\omega=\pi^{*} \Omega, \text { where } \pi: \mathcal{O}_{\xi_{0}} \rightarrow \mathcal{O}_{\xi_{1}} .
$$

$\Omega$ here is the canonical symplectic (surface) form of the 2 -sphere of radius $1 / \sqrt{2}$. Expressed by the group elements, $\Omega$ reads

$$
\Omega_{\zeta}=-d\left(\operatorname{Tr} \sigma_{3} \cdot g^{-1} d g\right), \quad\left(\zeta=\operatorname{Ad}_{g} \xi_{0}\right) .
$$

In 3-dimensional notation $\Omega$ is expressed cf. $[3,7,9]$.

$$
\left(1 / r^{3}\right) \varepsilon_{i j k} d x^{j} \otimes d x^{k}(r=|x|) \text {. }
$$

In these terms the Higgs charge (6.4) becomes

$$
m(\Phi)=(1 / 4 \pi) \int_{S^{2}} \operatorname{Tr}\left(\Phi\left[\partial_{\mu} \Phi, \partial_{\nu} \Phi\right]\right) d \sigma_{\mu \nu} .
$$

The orbit of $\xi_{1}$ is prequantizable: the integral of $\Omega$ on it is $2 \pi$.

The trace invariant or topological charge (Sect. 4) reads now

$$
I=-4 \pi \lambda m \text {. }
$$

The higher order invariants are also easily computed:

$$
I^{(n)}= \begin{cases}4 \pi(-1)^{n+1 / 2} \lambda^{n} m & \text { for } n \text { odd } \\ 0 & \text { for } n \text { even } .\end{cases}
$$

$\xi_{0}$ is automatically a $U(1)$ generator. The minimal generator parallel to it is

$$
\zeta_{0}=2 \xi_{1}=\sqrt{-1} \sigma_{3}=\sqrt{-1} \eta_{1} \text {. }
$$

The particles having an SU(2) internal structure belong to irreducible representations characterized by an integer $k$ (twice the isospin). Their electric charge reads hence, according to $(5.25)$,

$$
q=k \cdot q_{\min }=k \cdot\left(e_{0} / \sqrt{2}\right) .
$$


Similarly, the magnetic charge is obtained by (5.26) as

$$
g=m \cdot g_{\min }=m / \sqrt{2} e_{0}
$$

$2 q g=k m \in Z$, so Dirac's condition is satisfied.

$G=\mathrm{SU}(3)$. Let us consider now what happens for $G=\mathrm{SU}(3)$. The base point $\xi_{0}$ has now the form

$$
\xi_{0}=\sqrt{-1}\left[\begin{array}{lll}
\lambda_{1} & & \\
& \lambda_{2} & \\
& & \lambda_{3}
\end{array}\right], \lambda_{i} \in R, \sum_{i=1}^{N} \lambda_{i}=0 .
$$

There are two possibilities.

(i) Two of the $\lambda_{i}$ 's are equal. One can arrange, by applying a Weyl transformation, that $\lambda_{1}=\lambda_{2}$. By (2.29),

$$
H=\mathrm{S}\{\mathrm{U}(2) \times \mathrm{U}(1)\}=\mathrm{U}(2) \text {. }
$$

The orbit is the so-called minimal one. It has dimension 4 and has the topology of $P_{2}(C) . I=\{2\}$ so $Z(\mathfrak{h})$ is 1-dimensional and is generated by

$$
\xi_{2}=(\sqrt{-1} / 3)\left[\begin{array}{lll}
1 & & \\
& 1 & \\
& & -2
\end{array}\right]
$$

(or by $\xi_{0}$ which is proportional to $\xi_{2}$ ). The orbit of $\xi_{2}$ is also a minimal one and $\mathcal{O}_{\xi_{0}}$ projects obviously onto $\mathcal{O}_{\xi_{2}}$. The canonical symplectic structure of the latter is calculated the simplest way by representing the elements of $\mathrm{SU}(3)$ by elements of $C^{3}: \mathrm{SU}(3) \ni g=\left(Z_{1}, Z_{2}, Z_{3}\right)$, where $Z_{i} \in C^{3}, Z_{i} Z_{j}=\delta_{i j}, \operatorname{det} g=1$.

The pullback to $S U(3)$ of the canonical symplectic structure of $\mathcal{O}_{\xi_{2}}$ reads then

$$
\Omega^{(2)}=\sqrt{-1}\left(d Z_{1} \wedge d Z_{1}+d Z_{2} \wedge d Z_{2}\right) .
$$

There is again one Higgs charge, $m=m_{2}$, calculated as:

$$
m=(1 / 2 \pi \sqrt{-1}) \int_{S^{2}} \Phi^{*} \omega^{(2)}=(1 / 2 \pi \sqrt{-1}) \int_{S^{2}} g_{i}^{*} \Omega^{(2)} .
$$

( $g_{i}$ here is the lift of $\Phi$ on $U_{i}$. The result does not depend on the choice of the lift.)

The Trace invariant becomes

$$
I=-6 \pi \lambda m .
$$

Similarly, the higher order invariants are expressed as

$$
I^{(n)}=2 \pi \lambda^{n} \cdot(-1)^{(n+1) / 2}\left[1-(-2)^{n}\right] \cdot m .
$$

$\xi_{0}$ is parallel to $\xi_{2}=(1 / 3 \lambda) \xi_{0}$, so it generates a U(1). In fact,

$$
\mathfrak{h}=u(1)+\operatorname{su}(2) \text {. }
$$

The minimal generator $\zeta_{0}$ reads

$$
\zeta_{0}=3 \xi_{2}=\sqrt{-1}\left(\eta_{1}+2 \eta_{2}\right) \text {, }
$$


so $b_{2}=3$ and $n_{2}=2$. The electric charge of a particle belonging to an irreducible representation of $\mathrm{SU}(3)$ is a multiple of

$$
q_{\min }=e_{0} /\left|\zeta_{0}\right|=e_{0} / \sqrt{6}
$$

There is one basic magnetic charge, $g_{2}^{(0)}=g_{\min }$, and

$$
g_{2}=m \cdot g_{\min }=\sqrt{6} m / 4 e_{0} \text {. }
$$

The generalized Dirac condition becomes hence

$$
2 q_{\min } \cdot g_{\min }=1 / 2
$$

(the same result is obtained directly from (5.28) observing that $n_{2}=2$ ).

But there is also another possibility, namely when

(ii) all the $\lambda_{i}$ 's are different. $I=\{1,2\}$, yielding the residual symmetry group

$$
H=\mathrm{U}(1) \times \mathrm{U}(1) \text {. }
$$

The orbit of $\xi_{0}$ is 6-dimensional and has the topology of $\operatorname{PTP}_{2}(C)$. This is the maximal orbit.

$$
Z(\mathfrak{h})=\mathfrak{h}=\mathrm{u}(1)+\mathrm{u}(1)
$$

It is generated by $\xi_{2}$ and

$$
\xi_{1}=(\sqrt{-1} / 3)\left[\begin{array}{ccc}
2 & & \\
& -1 & \\
& & -1
\end{array}\right]
$$

The orbit of $\xi_{1}$ is again a minimal one. Its canonical symplectic structure is given by

$$
\Omega^{(1)}=\sqrt{-1} d Z_{1} \wedge d Z_{1}
$$

$\mathcal{O}_{\xi_{0}}$ projects now to both $\mathcal{O}_{\xi_{1}}$ and $\mathcal{O}_{\xi_{2}}$. There are two Higgs charges:

$$
m_{j}=(1 / 2 \pi \sqrt{-1}) \int_{S^{2}} \Phi^{*} \omega^{(j)}=(1 / 2 \pi \sqrt{-1}) \int_{S^{2}} g_{i}^{*} \Omega^{(j)}, \quad j=1,2 .
$$

$\left(g_{i}\right.$ denotes again the lift of $\Phi$ on $U_{i}$.) The $\omega^{(j)}$ 's are of course the pullbacks to $\mathcal{O}_{\xi_{0}}$ of the $\Omega^{(j)}$ 's. Both orbits $\mathcal{O}_{\xi_{j}}(j=1,2)$ are prequantizable.

The trace invariant becomes [11]

$$
I=-2 \pi\left\{\left(\lambda_{1}-\lambda_{2}\right) m_{1}+\left(2 \lambda_{2}+\lambda_{1}\right) m_{2}\right\} \text {. }
$$

For the higher order invariants our formulae yield

$$
I^{(n)}=2 \pi(-1)^{(n+1) / 2}\left[\left(\lambda_{1}^{n}-\lambda_{2}^{n}\right) m_{1}+\left(\lambda_{2}^{n}-\left\{-\lambda_{1}-\lambda_{2}\right\}^{n}\right) m_{2}\right] .
$$

$\xi_{0}$ generates a $\mathrm{U}(1)$ only if $\lambda_{1}$ and $\lambda_{2}$ are rationally related. In this case $n_{1}=r \lambda_{1}$ and $n_{2}-n_{1}=r \lambda_{2}$ are integers for a suitable real number $r$. Let us choose the least such positive $r$. Then

$$
r \xi_{0}-\zeta_{0}=\sqrt{-1}\left(n_{1} \eta_{1}+n_{2} \eta_{2}\right) .
$$

In terms of the $\xi_{i}$ 's $\zeta_{0}$ is expanded as

$$
\zeta_{0}=\left(2 n_{1}-n_{2}\right) \xi_{1}+\left(2 n_{2}-n_{1}\right) \xi_{2} .
$$


For the minimal electric charge we get from (5.10) (or (5.13)

$$
q_{\min }=e_{0} /\left|\zeta_{0}\right|=e_{0} / \sqrt{2}\left(n_{1}^{2}-n_{1} n_{2}+n_{2}^{2}\right) .
$$

On the other hand there are now two basic magnetic charges,

$$
g_{1}^{(0)}=g_{2}^{(0)}=\sqrt{6} / 4 e_{0} .
$$

The monopole's magnetic charge is hence

$$
g=\frac{m_{1}\left(2 n_{1}-n_{2}\right)+m_{2}\left(2 n_{2}-n_{1}\right)}{2 e_{0}\left|\zeta_{0}\right|}
$$

So the generalized Dirac conditions become

$$
2 q_{\min } g=\frac{n_{1}\left(2 m_{1}-m_{2}\right)+n_{2}\left(2 m_{2}-m_{1}\right)}{2\left(n_{1}^{2}-n_{1} n_{2}+n_{2}^{2}\right)} .
$$

$G=\mathrm{SU}(N)$. The generalization to $G=\mathrm{SU}(N)$ is now straightforward. Indeed, an element of $\mathrm{SU}(N)$ can be represented by $N$-tuples,

$$
g=\left\{\left(Z_{1}, \ldots, Z_{N}\right) \mid Z_{i} \in C^{N}, Z_{j} Z_{k}=\delta_{j k}, \operatorname{det}\left(Z_{1}, \ldots, Z_{N}\right)=1\right\} .
$$

The base point is given, after rearrangement, by (2.28) (see Sect. 2). The residual symmetry group is hence

$$
H=\mathrm{S}\left\{\mathrm{U}\left(i_{1}\right) \times \ldots \times \mathrm{U}\left(N-i_{p}\right)\right\} .
$$

The orbit is described as [24]

$$
\xi_{0}=\sqrt{-1} \sum_{i=1}^{N-1} \Lambda_{i}\left(Z_{i} Z_{i}-1 / N\right),
$$

where

$$
\Lambda_{i}=\lambda_{1}+\ldots+2 \lambda_{i}+\ldots+\lambda_{N-1} .
$$

$H$ has a $p$-dimensional centre whose Lie algebra is generated by those $\xi_{i_{k}}$ 's, $i_{k} \in I$ (cf. (2.23)). The corresponding orbits are prequantizable. Their canonical symplectic forms pull back to $G$ as

$$
\Omega^{\left(i_{k}\right)}=\sqrt{-1} \sum_{i=1}^{i_{k}} d Z_{j} \wedge d Z_{j}, \quad i_{k} \in I .
$$

The trace and respectively higher order invariants are obtained by (4.23) and (4.24),

$$
\operatorname{Tr}\left(\eta_{i_{k}}, \xi_{0}^{n}\right)=(-1)^{n}\left(\lambda_{i_{k}}^{n}-\lambda_{i_{k+1}}^{n}\right), \quad i_{k} \in I
$$

Consequently,

$$
I^{(n)}=2 \pi(-1)^{(n+1) / 2} \sum_{i_{k} \in I}\left(\lambda_{i_{k}}^{n}-\lambda_{i_{k+1}}^{n}\right) \cdot m_{k}
$$

Let us study finally the electromagnetic properties. First, $\xi_{0}$ generates a U(1) if the $\lambda_{i}$ 's in (2.28) are rationally related. If so, let us consider the minimal generator $\zeta_{0}$ 
parallel to it. Let $b_{k}(k=1, \ldots, p)$ and $n_{i} \in Z, i=1, \ldots, r$ its coefficients in its expansions in terms of the $\xi_{i_{k}}$ 's and the $\eta_{i}$ 's respectively. The formulae of Sect. 5 work beautifully: the minimal charge is expressed as

$$
q_{\min }=e_{0}\left\{\sum n_{i_{k}} b_{k}\right\}^{-1 / 2} .
$$

There are $p$ basic magnetic charges,

$$
g_{k}^{(0)}=1 / 2 e_{0}\left|\xi_{i_{k}}\right|=\left(1 / 2 e_{0}\right) \sqrt{N} /\left(N-i_{k}\right) .
$$

The generalized Dirac conditions are those given in (5.20) and (5.21) respectively.

In particular, if we choose [15],

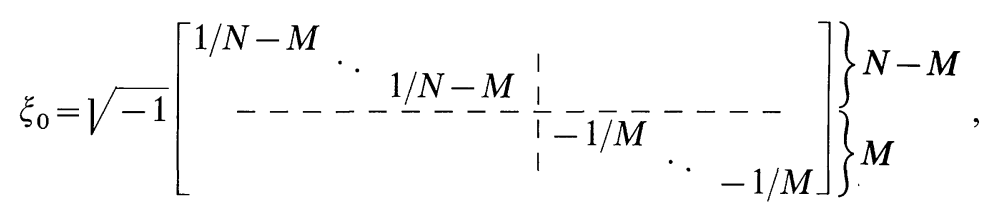

then there are just two blocks, $I=\{N-M\}$ and the 1-dimensional centre is generated by

$$
\xi_{N-M}=\{(N-M) \cdot M / N\} \xi_{0} .
$$

Being parallel to one of the fundamental $\xi_{i}$ 's, (6.48) generates a $U(1)$. The minimal generator in its direction is

$$
\zeta_{0}=n \xi_{0},
$$

where $n$ is the least common multiple of $(N-M)$ and $M$. This is obvious from (6.48). Alternatively, we can see this from the decomposition

$$
\left.\xi_{0}=\sqrt{-1}\left[\sum_{j=1}^{N-M}(j / N-M) \cdot \eta_{j}+\sum_{j=1}^{M-1}(M-j) / M\right) \cdot \eta_{N-M+j}\right] .
$$

This shows also that

$$
n_{N-M}=\mu_{N-M}\left(\zeta_{0}\right)=n .
$$

The minimal electric charge is hence

$$
q_{\min }=(1 / n) \sqrt{(N-M) M / N},
$$

while the basic magnetic charge is

$$
g_{\min }=g_{N-M}=(1 / 2) \sqrt{N /(N-M) M} .
$$

Thus the generalized Dirac condition becomes

$$
2 g_{\min } q_{\min }=1 / n .
$$

Acknowledgements. The authors are indebted to Prof. J.-M. Souriau for his interest in this work and for the hospitality extended to them in Marseille. They would also like to thank Profs. L.O'Raifeartaigh, L. Michel, and Drs. P.Forgács, Z. Horváth, and L.Palla for enlightening discussions. 


\section{References}

1. Tyupkin, Yu.S., Fateev, V.A., Shvarts, A.S.: Existence of heavy particles in gauge field theories. JETP. Lett. 21, 42 (1975)

2. Monastyrskii, M.I., Perelomov, A.: Concerning the existence of monopoles in gauge field theories. JETP. Lett. 21, 43 (1975)

3. Arafune, J., Freund, P.G.O., Goebel, C.J.: Topology of Higgs fields. J. Math. Phys. 16, 433 (1975)

4. Bogomolny, E.B.: The stability of classical solutions. Sovt. J. Nucl. Phys. 24, 449 (1976)

5. Manton, N.: Complex structure of monopoles. Nucl. Phys. B 135, 319 (1978)

6. Taubes, C.H.: Surface integrals and monopole charges in non-abelian gauge theories Commun. Math. Phys. 81, 299 (1981)

7. 't Hooft, G.: Magnetic monopoles in unified gauge theories. Nucl. Phys. B 79, 276 (1974)

8. Goddard, P., Olive, D.I.: Rep. Prog. Phys. 41, 1357 (1978)

9. Schwarz, A.S.: Magnetic monopoles in gauge theories. Nucl. Phys. B 112, 358 (1976)

10. Ward, R.: Deformations of the embedding of the SU(2) monopole solution in SU(3). Comm. Math. Phys. 86, 437 (1982)

11. Forgács, P., Horvath, Z., Palla, L.: On the construction of axially symmetric $\mathrm{SU}(N)$ monopoles. Nucl. Phys. B 221, 235 (1983)

12. Yang, C.N.: Charge quantization, compactness of the gauge group, and flux quantization. Phys. Dev. D 1, 2360 (1970)

13. Goddard, P., Olive, D.I.: Charge quantization in theories with an adjoint representation Higgs mechanism. Nucl. Phys. B 191, 511 (1981)

14. Corrigan, E., Olive, D.I.: Colour and magnetic monopoles. Nucl. Phys. B 110, 237 (1976)

15. Horváth, Z., Palla, L.: Monopoles and grand unification theories. Phys. Lett. 69B, 197 (1977)

16. Humphreys, J.E.: Introduction to Lie algebras and representation theory. Berlin, Heidelberg, New York: Springer 1972

17. Wallach, N.R.: Harmonic analysis on homogeneous spaces. New York: Dekker 1973

18. Coleman, S.: Classical lumps and their quantum Descendents. In: New phenomena in subnuclear physics, Zichichi, A. (ed.). New York: Plenum Press 1977

19. Kobayashi, S., Nomizu, K.: Foundations of differential geometry. Vol. I and Vol. II. New York: Interscience 1963 and 1969

20. Goddard, P., Nuyts, J., Olive, D.: Gauge theories and magnetic charge. Nucl. Phys. B 125, 1 (1977)

21. Kirillov, A.: Eléments de la théorie des représentations. Moscou: MIR 1974

22. Kostant, B.: Quantization and Unitary representations. In: Lecture Notes in Mathematics, Vol. 170. Berlin, Heidelberg, New York: Springer 1970

23. Souriau, J.-M.: Structure des systèmes dynamiques. Paris: Dunod 1970

24. Duval, C.: On the polarizers of compact sem-simple Lie groups. Applications. Ann. Inst. H. Poincaré 34, 95 (1981)

25. Prasad, M.K., Sommerfield, C.M.: Exact classical solution for the 't Hooft monopole and the Julia-Zee dyon. Phys. Rev. Lett. 35, 760 (1975)

26. Lichtenberg, D.B.: Unitary symmetry and elementary particles. $2^{\text {nd }}$ edn. New York: Academic Press 1978

27. Weinberg, E.J.: Nucl. Phys. B 167, 500 (1980)

28. Weinberg, E.J.: Nucl. Phys. B 203, 445 (1982)

Communicated by A. Jaffe

Received June 4, 1984 
